\title{
Molecular flexibility of Mycobacterium tuberculosis ribosome recycling factor and its functional consequences: An exploration involving mutants
}

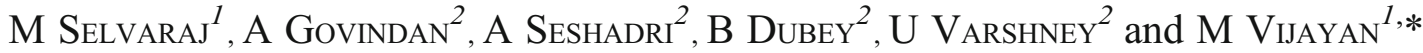 \\ ${ }^{1}$ Molecular Biophysics Unit, ${ }^{2}$ Department of Microbiology and Cell Biology, Indian Institute of \\ Science, Bangalore 560 012, India \\ *Corresponding author (Fax, +91-80-2360-0535, +91-80-2360-0683; \\ Email,mv@mbu.iisc.ernet.in)
}

\begin{abstract}
Internal mobility of the two domain molecule of ribosome recycling factor (RRF) is known to be important for its action. Mycobacterium tuberculosis RRF does not complement E. coli for its deficiency of RRF (in the presence of E. coli EF-G alone). Crystal structure had revealed higher rigidity of the M. tuberculosis RRF due to the presence of additional salt bridges between domains. Two inter-domain salt bridges and one between the linker region and the domain containing C-terminal residues were disrupted by appropriate mutations. Except for a C-terminal deletion mutant, all mutants showed RRF activity in E. coli when M. tuberculosis EF-G was also co-expressed. The crystal structures of the point mutants, that of the $\mathrm{C}$-terminal deletion mutant and that of the protein grown in the presence of a detergent, were determined. The increased mobility resulting from the disruption of the salt bridge involving the hinge region allows the appropriate mutant to weakly complement $E$. coli for its deficiency of RRF even in the absence of simultaneous expression of the mycobacterial EF-G. The loss of activity of the C-terminal deletion mutant appears to be partly due to the rigidification of the molecule consequent to changes in the hinge region.
\end{abstract}

[Selvaraj M, Govindan A, Seshadri A, Dubey B, Varshney U and Vijayan M 2013 Molecular flexibility of Mycobacterium tuberculosis ribosome recycling factor and its functional consequences: An exploration involving mutants. J. Biosci. 38 845-855] DOI 10.1007/s12038-013-9381-0

\section{Introduction}

At the step of translation termination, the action of class I (RF1/RF2) and class II (RF3) release factors leads to the release of the polypeptide chains from the translating ribosomes. However, the ribosomes themselves and the deacylated tRNA(s) remain bound to the mRNA in a posttermination complex. For the ribosomal subunits to take part in the next round of translation, the post-termination complexes are dissociated by the combined action of the ribosome recycling factor (RRF) and elongation factor $G(E F-G)$ (Hirashima and Kaji 1972; Seshadri and Varshney 2006). Both the RRF and EF-G are essential proteins for the survival of Escherichia coli. Biochemical and genetic studies from our laboratory using heterologous factors have revealed that specific interactions between RRF and EFG are important for ribosome recycling. We have previously shown that RRF from M. tuberculosis (MtRRF) is unable to rescue an E. coli strain carrying a temperature sensitive allele of RRF $\left(f r r^{t s}\right)$ at the non-permissive temperature. However, when EF-G from M. tuberculosis (Mt-EFG) is also coexpressed, it rescues the strain for its temperature sensitive phenotype, showing that there exist specific interactions between the two molecules and that these interactions are essential for ribosome recycling (Rao and Varshney 2001; Seshadri et al. 2010).

Ribosome recycling has been subjected to extensive biochemical and structural studies (Hirokawa et al. 2006). The RRF molecule has an overall L-shape made up of two domains (Selmer et al. 1999). The N-terminal and C-terminal stretches together form the helix bundle domain 1 , while the polypeptide in between constitute domain 2 with

Keywords. Activity and complementation; molecular libration; mutational analysis; plasticity and protein function; protein structure; ribosome disassembly 
an $\alpha / \beta$ fold. The two domains are connected by a linker region (hinge) made up of two short polypeptide stretches. The mobility of the L-shaped RRF molecule from different sources, has received particular attention. The structure of MtRRF was determined earlier (Saikrishnan et al. 2005) as part of a long range effort on mycobacterial proteins in this laboratory (Vijayan 2005; Prabu et al. 2009; Krishna et al. 2007; Selvaraj et al. 2007; Kaushal et al. 2008; Roy et al. 2008; Chetnani et al. 2010) and elsewhere (Terwilliger et al. 2003; Arora et al. 2011). In the structure, domain 1 consists of residues 1-26 and 107-185 and domain 2 consists of residues 34-102 (Saikrishnan et al. 2005). Residues 27-33 and 103-106 constitute linkers 1 and 2, respectively. The different components of the molecular mobility of RRF have been characterized in detail in our earlier studies. In this context, the inability of $M t R R F$ to complement $E$. coli for its deficiency of RRF ( $E c R R F$ ), at least in part, appeared to be related to the comparative rigidity of the mycobacterial protein. The domains in MtRRF could be connected by two salt bridges instead of the one in $E c R R F$. Furthermore, a salt bridge involving a residue in the hinge region also appeared to have an important structural role. In the work reported here, these salt bridges were disrupted through appropriate mutations, the structures of the mutants were determined and their activities and their ability to complement $E$. coli for its deficiency of RRF were examined. Also studied is a C-terminal deletion mutant of MtRRF, which is known to be inactive. Following the cue from structural studies on EcRRF (Kim et al. 2000), interaction of a detergent, (decyl- $\beta$-D-maltopyranoside) with $M t R R F$ also has been explored. The crystallographic and biochemical results presented here provide valuable insights into the structural basis for the different components of the mobility of the molecule and the role of molecular plasticity in the action of RRF.

\section{Methods}

\subsection{Antibiotics, chemicals and strains}

Components of LB agar or broth were purchased from Difco. All chemicals were from Sigma unless specified. IPTG was from Calbiochem. TG1 cells were used for all cloning purposes and were grown at $37^{\circ} \mathrm{C}$. LJ14 cells were grown at $30^{\circ} \mathrm{C}$ wherever not specified.

\subsection{Generation of mutant $R R F$}

Mutants of $M t R R F$ were generated using previously generated constructs as templates. The R31A and R109 mutants were generated using pET11dMtRRF, as template. The R39G mutant was generated using $\mathrm{p} T \mathrm{rc} M t \mathrm{RRF}$ as template. The R39G R109A double mutant was generated using the
R39G mutant construct as template. All mutants were generated by site-directed mutagenesis using an inverse PCRbased approach using complementary pairs of primers (see table 1 for sequences). The reaction mixture consisted of around $100 \mathrm{ng}$ of template DNA, $10 \mathrm{pmol}$ each of the forward and the reverse primer, $250 \mu \mathrm{M}$ dNTPs, 0.4 units of Phusion DNA polymerase (ThermoScientific) and the manufacturer supplied buffer (5X HF buffer) diluted to $1 \mathrm{X}$ concentration. The cycling conditions were as follows: $98^{\circ} \mathrm{C}$ for $1 \mathrm{~min}, 45^{\circ} \mathrm{C}$ for $1 \mathrm{~min}, 72^{\circ} \mathrm{C}$ for $6 \mathrm{~min}$. Amplification was done for 18 cycles followed by a final extension of $10 \mathrm{~min}$ at $72^{\circ} \mathrm{C}$. The PCR mixture was digested with 10 units of DpnI overnight and $10 \mu \mathrm{L}$ of the mixture was transformed into TG1 competent cells and selected on ampicillin. Mutants were confirmed by sequencing. The $p \operatorname{Trc}$ or pET constructs of the mutants obtained were digested with NcoI and HindIII to release the RRF gene and this fragment was subcloned into a similarly digested pACDH plasmid to generate pACDHMtRRF mutants which were used for complementation assays. Cloning of MtEF-G into pTrc99c has been previously described (Rao and Varshney 2001).

\subsection{Complementation assays}

For checking the activity of mutant RRF's with $M t$ EF-G, E. coli LJ14 $\left(f r r^{t s}\right)$ cells were transformed with pTrcMtEF-G and $\mathrm{pACDH} M t \mathrm{RRF}$ mutants. Independent colonies were grown to saturation at $30^{\circ} \mathrm{C}$, streaked out on $\mathrm{LB}$ agar plates containing either ampicillin $(100 \mu \mathrm{g} / \mathrm{mL})$ and tetracycline $(7.5 \mu \mathrm{g} / \mathrm{mL})$ or ampicillin, tetracycline and $0.5 \mathrm{mM}$ IPTG and grown at $30^{\circ} \mathrm{C}, 39^{\circ} \mathrm{C}$ and $42^{\circ} \mathrm{C}$. For checking the ability of the mutants to complement the $\mathrm{rrr}^{\text {ts }}$ phenotype, LJ14 cells were transformed with pACDHMtRRF mutants and independent colonies were grown to saturation at $30^{\circ} \mathrm{C}$. Growth curves were constructed out using at least three replicates of each culture. Overnight cultures grown at $30^{\circ} \mathrm{C}$ were diluted 100 -fold in LB containing tetracycline. $200 \mu \mathrm{L}$ aliquots of the diluted culture were taken in honeycomb plates and shaken in an automated Bioscreen $\mathrm{C}$ growth reader (Oy Growth) maintained at $39^{\circ} \mathrm{C} . \mathrm{OD}_{600}$ was measured at $1 \mathrm{~h}$ intervals, and data were plotted with standard errors.

\subsection{Protein purification and crystallization}

Mutant RRFs were purified as described before (Saikrishnan et al. 2004). Crystals used in the present analysis were grown in conditions close to that in which wild-type crystals grew (Saikrishnan et al. 2004). Briefly, a solution containing 6\% PEG 4 K, 20 mM Tris-HCl (pH 7.8), 0.6 mM Cadmium acetate and 5\% PEG 400 was mixed with a protein sample of $10 \mathrm{mg} / \mathrm{ml}$ concentration at equal volume. Crystals grew in two weeks in micro batch crystallization set up under a layer 
Table 1. Plasmids, strains and primers used for generation of mutants and complementation

\begin{tabular}{llc}
\hline Primer/Strain/Plasmid & \multicolumn{1}{c}{ Relevant details } & Reference \\
\hline R31A-Fp & 5'-CTATCCGTACCGGCGCAGCCAACCCTGGCA-3' & This work \\
R31A-Rp & 5'-TGCCAGGGTTGGCTGCGCCGGTACGGATAG-3' & This work \\
R109A-Fp & 5'-AGCTCACCGAAGAAGCACGGCGAGAGCTGGT-3' & This work \\
R109A-Rp & 5'-ACCAGCTCTCGCCGTGCTTCTTCGGTGAGCT-3' & This work \\
R39G-Fp & 5'-CATGTTCTCTGGGATCACCATCG-3' & This work \\
R39G-Rp & 5'-CGATGGTGATCCCAGAGAACATG-3' & This work \\
pACDH & Plasmid with pACYC origin of replication, Tet ${ }^{\mathrm{R}}$ & Rao and Varshney 2002 \\
LJ14 & MC1061 containing the frr14 ${ }^{\text {ss }}$ allele & Janosi et al. 1998 \\
\hline
\end{tabular}

of paraffin oil and silicon oil mixed in equal ratio. $2 \mathrm{mM}$ detergent was included in the solutions in soaking studies. All crystals had nearly the same morphology as that of the wild-type crystals.

\subsection{Structure determination and analysis}

The X-ray data were collected at $100 \mathrm{~K}$ using a $345 \mathrm{~mm}$ MAR imaging plate mounted on a Rigaku RU-200 X-ray generator, with a rotation of $1^{\circ}$ per frame. The images were processed and scaled by use of MOSFLM and SCALA in the CCP4 program suite (Winn et al. 2011). The wild-type MtRRF structure was used as the initial model in all the cases where the cell dimension and space group were the same, with appropriate residue changes. In the case of $\Delta \mathrm{C} 6 M t \mathrm{RRF}$ and R31AMtRRF, domain 1 and domain 2 of RRF were searched separately using PHASER (Storoni et al. 2004). The structures were initially refined using CNS (Brunger et al. 1998). REFMAC in CCP4 was used in the subsequent stages. Different steps in the process were rigid body refinement, simulated annealing, positional refinement and $\mathrm{B}$ factor refinement. The TLS refinement option in REFMAC was employed in the final cycles with the three helices in domain I and domain II as rigid groups. Bulk solvent correction was used in all the cases. Model building was performed using COOT (Emsley and Cowtan 2004). Water molecules were added, wherever the peak height in the 2FoFc map is greater than 0.8 and the FoFc map greater than 2.5. The crystallographic data and refinement parameters are given in table 2 .

\section{Results}

\subsection{General considerations}

Three structure determinations of $M t \mathrm{RRF}$ have been carried out earlier in our laboratory, one at low temperature at $2.1 \AA$ resolution and the other two at room temperature at resolutions of $2.6 \AA$ and $2.9 \AA$ (Saikrishnan et al. 2005). Three salt bridges in them are of particular interests in relation to the situation in $E c$ RRF (figure 1). Two structures of $E c R R F$ are available. One of them has a detergent molecule (decyl- $\beta$-Dmaltopyranoside) lodged between the domains (Kim et al. 2000) and the molecular structure is far too distorted to be of use in a general discussion. The structure of a point mutant (R132GEcRRF) of the protein is also available (Nakano et al. 2002). The mutation does not appear to have any structural effect on molecular conformation, particularly inter domain orientation, and hence the mutant structure is used here for comparison as representative $E c$ RRF. The three salt bridges in $M t$ RRF referred to earlier are R39..D23 and R109..D86, which connect the two domains, and R31...E184, which connects the hinge with the second domain (table 3 ). The N...O distances in the first and the third are appropriate for hydrogen bonded salt bridges in the low temperature (LT) structure, which has been used for comparisons. The corresponding distance is somewhat high in the second salt bridge. However, the second salt bridge with an appropriate distance was observed in a room temperature structure and was therefore included in the discussion (Saikrishnan et al. 2005). The first salt bridge cannot occur in EcRRF as the residues at 39 and 23 in it are glycine and glutamine respectively. Residues 109 and 86 remain $R$ and $D$ respectively, but the distance between them is too high to permit the formation of a salt bridge. Residue 184 is glutamine in $E c$ RRF. However, $31 \mathrm{R}$ forms a salt bridge with $181 \mathrm{E}$. This is the only one out of the three, present in EcRRF. In terms of distances, even this appears to be weaker than the $\mathrm{R} 31 \ldots 184 \mathrm{E}$ salt bridge in MtRRF. It was therefore hypothesized that the higher rigidity of $M t R R F$ consequent of the presence of the salt bridges could, at least in part, contribute to its inability to complement $E$. coli for its deficiency of RRF (i.e. when mycobacterial EF-G is not co-expressed). To test this hypothesis, three point mutants R39G (R39GMtRRF), R109A (R109AMtRRF) and R31A (R31AMtRRF) and the double mutant R39G.R109A (R39G.R109AMtRRF) were prepared and studied. Earlier studies had indicated the crucial role of the C-terminal residues in the action of RRF (Toyoda et al. 2000; Rao and Varshney 2002; Fujiwara et al. 1999). The structure of a C-terminal deletion mutant of $M t \mathrm{RRF}(\triangle \mathrm{C} 6 M t \mathrm{RRF})$ was studied to explore 
Table 2. Data collection and refinement statistics

\begin{tabular}{|c|c|c|c|c|c|}
\hline & $\begin{array}{l}\text { R39GMtRRF } \\
(4 \mathrm{KAW})\end{array}$ & $\begin{array}{l}\mathrm{R} 109 \mathrm{~A} M t \mathrm{RRF} \\
(4 \mathrm{~KB} 2)\end{array}$ & $\begin{array}{l}\mathrm{R} 31 \mathrm{~A} M t \mathrm{RRF} \\
(4 \mathrm{~KB} 4)\end{array}$ & $\begin{array}{l}\Delta \mathrm{C} 6 M t \mathrm{RRF} \\
(4 \mathrm{KC} 6)\end{array}$ & $\begin{array}{l}\text { Wild-type } M t \text { RRF } \\
\text { with detergent } \\
\text { (4KDD) }\end{array}$ \\
\hline Space group & P21 & $\mathrm{P} 21$ & $\mathrm{C} 2$ & P212121 & $\mathrm{P} 21$ \\
\hline $\begin{array}{l}\text { Unit cell parameter } \\
\text { a,b,c }(\AA) \beta\left(^{\circ}\right)\end{array}$ & $\begin{array}{l}56.3,33.6 \\
\quad 60.8,122.8\end{array}$ & $56.5,33.61 .1,113.2$ & $\begin{array}{l}95.2,40.5 \\
\quad 68.5,118.4\end{array}$ & $40.9,48.8,84.0$ & $\begin{array}{l}56.3,33.7 \\
\quad 61.5,112.8\end{array}$ \\
\hline Resolution $(\AA)$ & $2.5(2.64-2.50)$ & $2.3(2.42-2.30)$ & $2.25(2.37-2.25)$ & $2.4(2.53-2.40)$ & $1.9(2.00-1.90)$ \\
\hline Temperature & $100 \mathrm{~K}$ & $100 \mathrm{~K}$ & $100 \mathrm{~K}$ & $100 \mathrm{~K}$ & $100 \mathrm{~K}$ \\
\hline $\mathrm{Vm}$ & 2.6 & 2.6 & 2.9 & 2.1 & 2.7 \\
\hline Solvent content $(\%)$ & 53.7 & 54 & 57 & 41.5 & 55.7 \\
\hline Unique reflections & $7487(1085)$ & $9680(1394)$ & $11114(1601)$ & 7009 (987) & $17141(2485)$ \\
\hline Multiplicity & $2.9(2.9)$ & $4(4)$ & 4 & $4.6(4.6)$ & $3.9(3.8)$ \\
\hline Completeness (\%) & $99.6(100.0)$ & $99.7(98.9)$ & $100(100.0)$ & $99.9(100.0)$ & $99.9(100.0)$ \\
\hline Mean $\mathrm{I} / \sigma(\mathrm{I})$ & $7.7(2.3)$ & $6.9(2.5)$ & $12.5(3.2)$ & $8.2(2.2)$ & $7.2(2.1)$ \\
\hline $\mathrm{R}_{\mathrm{sym}} \dagger$ & $14.4(52.3)$ & $16.5(53.6)$ & $8.7(39.1)$ & $12.4(64.1)$ & $13.3(60.1)$ \\
\hline \multicolumn{6}{|l|}{ Refinement and model statistics ${ }^{\S}$} \\
\hline Rfactor $(\%)$ & 23.4 & 22.6 & 21.5 & 25.0 & 24.9 \\
\hline Rfree $(\%)$ & 27.3 & 26.6 & 26.3 & 30.3 & 27.0 \\
\hline \multicolumn{6}{|l|}{ R.m.s. deviation from ideal } \\
\hline Bonds $(\AA)$ & 0.007 & 0.009 & 0.007 & 0.006 & 0.007 \\
\hline Angles $\left(^{\circ}\right)$ & 1.0 & 1.2 & 0.9 & 1.1 & 1.1 \\
\hline \multicolumn{6}{|l|}{ Ramachandran plot statistics\# } \\
\hline Favoured regions & 92.1 & 93.4 & 92.9 & 85.9 & 93.4 \\
\hline Additionally allowed regions & 7.3 & 6.0 & 5.4 & 12.9 & 6.6 \\
\hline Generouslly allowed region & 0.6 & 0.6 & 0.6 & 1.2 & - \\
\hline Disallowed regions & - & - & 1.2 & - & - \\
\hline Residues built in the protein & 183 & 184 & 184 & 178 & 184 \\
\hline No. of water molecules & 89 & 102 & 86 & 131 & 197 \\
\hline
\end{tabular}

${ }^{\dagger} R_{\text {sym }}=\sum_{\mathrm{h}} \sum / / h_{\mathrm{h}}-</ \mathrm{h}>/ \sum_{\mathrm{h}} \sum /</ \mathrm{h}>$, where $/ /$ is the $/$ th observation of reflection $\mathbf{h}$ and $</ \mathrm{h}>$ is the weighted average intensity for all observation / of reflection $\mathbf{h}$.

\# Calculated for non-glycine and non-proline residues using PROCHECK.

Values in parenthesis corresponds to the highest resolution shell.

the dependence of their role in the mobility of the molecule. In the light of the information on the known structure of EcRRF in complex with the detergent (Kim et al. 2000), structures of MtRRF crystals grown in the presence of the detergent were also analysed.

\subsection{Complementation analysis}

The gene encoding EF-G is essential in E. coli and thus cannot be knocked out without the support of another EF-G (Seshadri and Varshney 2006; Seshadri et al. 2010). Activity of all the generated RRF mutants was checked in vivo, by co-transforming $M t \mathrm{EF}-\mathrm{G}$ and $M t \mathrm{RRF}$ mutants into E. coli LJ14. Figure 2 shows the results of this activity assay. Among the point mutants, all were active in the presence of
$M t$ EF-G and rescued the $f r r^{t s}$ strain of $E$. coli LJ14, both at semi-permissive $\left(39^{\circ} \mathrm{C}\right)$ and non-permissive $\left(42^{\circ} \mathrm{C}\right)$ temperatures (figure $2 \mathrm{~b}$ and $\mathrm{c}$, sectors 4 to 9), suggesting that the point mutations do not adversely affect the function of $M t$ RRF mutants. In contrast, the double mutant R39G.R109AMtRRF showed a compromised phenotype in rescuing the LJ14 strain, compared to other point mutants (figure $2 \mathrm{~b}$ and $\mathrm{c}$, sectors 10 and 11). It has already been shown that $\triangle \mathrm{C} 6 M t \mathrm{RRF}$ is inactive (Rao and Varshney 2002). However, in the absence of simultaneous expression of $M t$ EF-G, neither the wild-type nor the mutants of $M t \mathrm{RRF}$ could complement the frr ${ }^{\text {ts }}$ phenotype of E.coli LJ14 at the non-permissive temperature $\left(42^{\circ} \mathrm{C}\right)$, indicating their inability to function efficiently with $E c$ EF-G. However, a weak complementation could be observed with R31AMtRRF (in which the mutation involves the hinge region) at semi- 

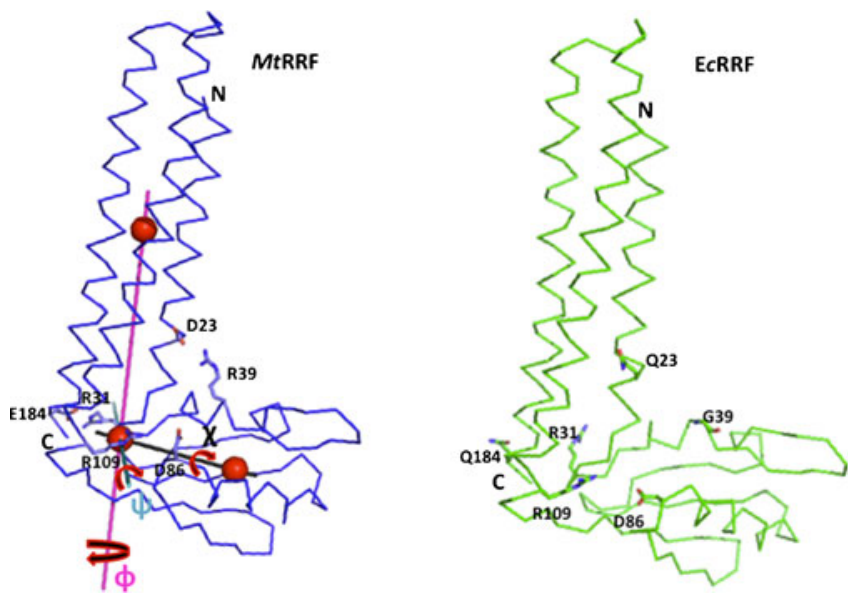

Figure 1. The structures of $M t R R F$ and $E c R R F$. The rotations used for describing the mobility of the molecule and the residues involved in salt bridges in MtRRF are shown. The corresponding residues in $E c R R F$ are also indicated.

permissive temperature $\left(39^{\circ} \mathrm{C}\right)$ compared to wild-type and other mutants of $M t R R F$ (figure 3). To sum up, with $M t$ EF-G, all single point mutants retain activity, the double mutant R39G.R109AMtRRF shows a weak activity and $\Delta \mathrm{C} 6 M t \mathrm{RRF}$ shows no activity. Interestingly,
R31AMtRRF shows a gain of weak activity/complementation in E. coli LJ14 with EcEF-G. The other mutants and the wild-type protein show no activity/complementation with EcEFG.

\subsection{Overall features of crystal structures}

The crystal structures of R39GMtRRF, R109AMtRRF, $\mathrm{R} 31 \mathrm{~A} M t \mathrm{RRF}$ and $\triangle \mathrm{C} 6 M t \mathrm{RRF}$ have been determined at a resolutions from $2.25 \AA$ to $2.5 \AA$ (table 2). The crystals of R39GMtRRF and R109AMtRRF are monoclinic and isomorphous to the wild-type crystals reported earlier. Those of R31AMtRRF are also monoclinic but non-isomorphous to the wild-type crystals (Saikrishnan et al. 2004). Those of $\Delta \mathrm{C} 6 M t \mathrm{RRF}$ are orthorhombic. The structure of $M t R R F$ in crystals grown in the presence of the detergent decyl- $\beta$-D-maltopyranoside $(1.9 \AA$ resolution) was also determined.

As in the case of the proteins from other bacterial and archeal sources, $M t$ RRF has a L-shaped molecule containing two domains and a linker region (figure 4). The $\mathrm{N}$-terminal and C-terminal stretches involving residues 2-26 and 107185 form domain 1 with a three-helix bundle structure. Domain 2 with an $\alpha / \beta$ structure is made up of residues 34 102. Peptide stretches $27-33$ and 103-106 constitute the linker region. The overall mobility of the protein can be

Table 3. Distances of $5 \AA$ or less between appropriate atoms in residues involved in salt bridges

\begin{tabular}{|c|c|c|c|c|c|c|c|}
\hline \multirow{2}{*}{ S. No. } & \multirow{2}{*}{$\frac{\text { RRF structure }}{\text { Wild-type } M t \text { RRF (Low temperature) }}$} & \multicolumn{2}{|l|}{ R39...D23 (Å) } & \multicolumn{2}{|c|}{ R109...D86 (Å) } & \multicolumn{2}{|c|}{ R31...E184 (Å) } \\
\hline & & NH1 ...OD2 & 3.5 & NH2 ...OD1 & 4.7 & NH2 ...OE1 & 4.5 \\
\hline & & NH2 ...OD1 & 4.9 & $\mathrm{NH} 2 \ldots \mathrm{OD} 2$ & 5.0 & NE...OE1 & 2.8 \\
\hline & & $\mathrm{NH} 2 \ldots \mathrm{OD} 2$ & 3.0 & & & NH1 ...OE2 & 4.9 \\
\hline & & NE...OD2 & 5.0 & & & $\mathrm{NH} 2 \ldots \mathrm{OD} 2$ & 3.3 \\
\hline \multirow[t]{5}{*}{2} & R39GMtRRF & - & & NH2 ...OD1 & 3.9 & NE...OE1 & 4.8 \\
\hline & & & & NH2 ...OD2 & 4.7 & NH1 ...OE2 & 4.8 \\
\hline & & & & & & NH2 ...OE2 & 3.7 \\
\hline & & & & & & NE...OE2 & 2.7 \\
\hline & & & & & & NE...OE2 & 2.6 \\
\hline \multirow[t]{3}{*}{3} & $\mathrm{R} 109 \mathrm{~A} M t \mathrm{RRF}$ & NH1 ...OD1 & 4.6 & - & & NH1 ...OE2 & 4.9 \\
\hline & & NH2 ...OD1 & 3.1 & & & NH2 ...OE2 & 3.7 \\
\hline & & & & & & NE...OE2 & 5.0 \\
\hline 4 & $\mathrm{R} 31 \mathrm{~A} M t \mathrm{RRF}$ & - & & - & & - & \\
\hline 5 & $\Delta \mathrm{C} 6 M t \mathrm{RRF}$ & NH1 ...OD2 & 3.4 & - & & - & \\
\hline & & NE...OD2 & 4.9 & & & & \\
\hline \multirow[t]{4}{*}{6} & MtRRF.detergent & NH2 ...OD1 & 3.7 & NH2 ...OD1 & 3.7 & NH2 ...OE1 & 3.1 \\
\hline & & $\mathrm{NH} 2 \ldots \mathrm{OD} 2$ & 4.3 & NH2 ...OD2 & 4.4 & NE...OE1 & 4.7 \\
\hline & & & & & & $\mathrm{NH} 2 \ldots \mathrm{OE} 2$ & 3.5 \\
\hline & & & & & & NE...OD2 & 3.8 \\
\hline
\end{tabular}

Distances of $4 \AA$ or less are in bold. 


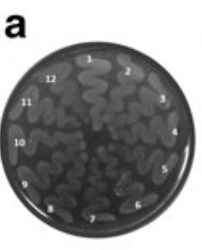

$30^{\circ} \mathrm{C}$

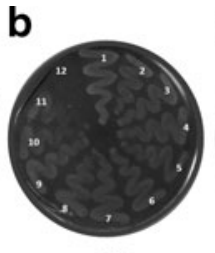

$39^{\circ} \mathrm{C}$

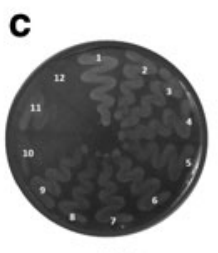

$42^{\circ} \mathrm{C}$
Figure 2. Analysis of activity of $M t R R F$ mutants in the presence of $M t \mathrm{EF}-\mathrm{G}$ in the $\mathrm{frr}^{t s} \mathrm{LJ} 14$ strain of $E$. coli. All strains are grown on Amp Tet agar plates supplemented with $0.5 \mathrm{mM}$ IPTG. Vector control (LJ14pTrc MtEF-G+ pACDH) is shown in sector 12 .

described in terms of the angle between the two arms of the molecule $(\psi)$, the rotation of domain 2 in the plane nearly perpendicular to the axis of the helix bundle which constitute domain $1(\varphi)$ and the rotation of domain 2 about an axis which is nearly parallel to its principal axis and passes through the centre of mass of the domain $(\chi)$ (Saikrishnan et al. 2005) (figure 1). In the case of $\varphi$ and $\chi$, the values found in the low temperature structure of $M t R R F$ $(1 \mathrm{WQG})$ are taken as reference points and designated as zero. The values of these angles in the five structures reported here are given in table 4 .

\subsection{Crystal structures of R39GMtRRF and R109AMtRRF}

It turns out that the structure of the R39GMtRRF mutant remains essentially the same as that of the wild-type protein, with variations of $2^{\circ}$ in $\psi$ and less than $4^{\circ}$ each in the in $\varphi$ and $\chi$ (table 4). The r.m.s. deviations in C $\alpha$ positions on domainwise superposition are also less than $0.3 \AA$ and

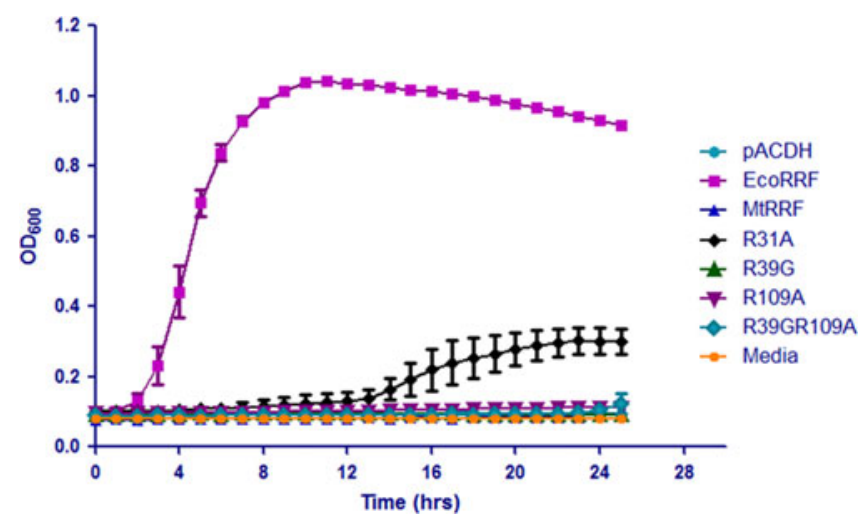

Figure 3. Analysis of complementation of the $f r r^{t s}$ LJ14 strain of E.coli by MtRRF mutants. Vector control (pACDH) is shown in light blue circles. a

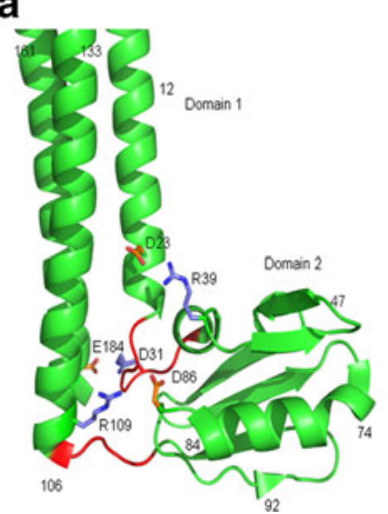

b

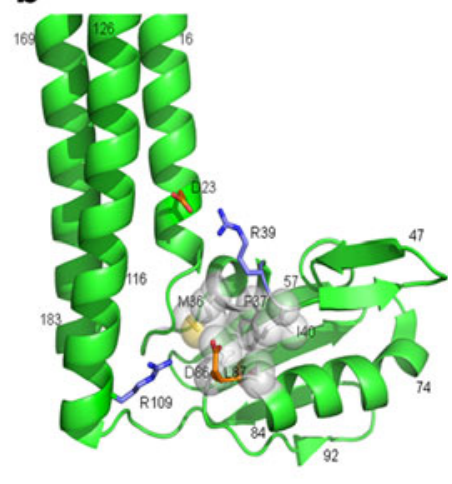

Figure 4. (a) Overall structure of $M t R R F$ and the locations of the three salt bridges. Side chains of arginine are in blue and aspartic, glutamic acids are in orange. (b) Hydrophobic cluster connecting R39 and D86.

$0.4 \AA$ for domain 1 and domain 2, respectively, with respect to wild-type $M t$ RRF structure (1WQG). However, the closest distance between a nitrogen atom in the guanidyl group of $\operatorname{Arg} 109$ and a carboxylate oxygen in Asp86 is $3.9 \AA$ in the mutant, whereas it is $4.7 \AA$ in the comparable native structure in which there is a strong salt bridge between Arg39 and Asp23 (figure 4a and table 3). Thus, there appears to be a complementarity in the roles of the two salt bridges. In the absence of R39...D23, the R109...D86 interactions tends to become strong. The communication between the two could be through a continuous hydrophobic cluster between Arg39 and Asp86 in domain 2 (figure $4 \mathrm{~b}$ ). The crystal structure of R109AMtRRF is again similar to that of $M t \mathrm{RRF}$ in terms of cell dimensions, molecular shape and structure. The structure opens by $6^{\circ}$ with respect to wild-type in $\psi$ and retains nearly the same $\varphi$ and $\chi$. The close similarity of the structures of the two mutants with that of the wild-type protein is reflected in the similar behavior of the three in relation to activity and complementation.

Table 4. Movement of domain 2 quantified as $\psi, \varphi$ and $\chi$

\begin{tabular}{lccc}
\hline Structure & $\psi$ (degrees) & $\varphi$ (degrees) & $\chi$ (degrees) \\
\hline$M t$ RRF & 96 & 0 & 0 \\
R39GMtRRF & 98 & -3 & 3 \\
R109AMtRRF & 102 & 0 & 0 \\
R31AMtRRF & 85 & -20 & 35 \\
$\Delta$ C6MtRRF & 83 & 0 & 24 \\
$M t$ RRF. detergent & 99 & -2 & 2
\end{tabular}




\subsection{Structures of R31AMtRRF and $\triangle \mathrm{C} 6 \mathrm{MtRRF}$}

The structure of R31AMtRRF differs from the wild-type structure and those of the other two point mutants of MtRRF. Abolition of the R31...E184 interaction in R31A results in an upward movement of domain 2 , with an acute $\psi$ angle of $85^{\circ}$. The mutation also results in the movement of domain 2 by about $-20^{\circ}$ in $\varphi$ and $35^{\circ}$ in $\chi$. Thus, R31A mutation results in a major domain reorganization, indicating the important role of the R31...E184 interaction in maintaining the correct domain orientation. Interdomain salt bridges do not occur in the R31A structure. Thus, R31A appears to have a more relaxed and flexible structure than the other two point mutants and the wild-type molecule.

The C-terminal region of RRF forms part of domain I. $E c$ RRF retains its activity even up to a deletion of seven residues at the $\mathrm{C}$-terminus, but loses its function when nine or more residues are deleted (Fujiwara et al. 1999). $\Delta \mathrm{C} 6 M t \mathrm{RRF}$ completely lacks ribosome recycling activity and has a compromised binding to ribosome, although CD results indicate that it folds as the wild-type protein does (Rao and Varshney 2002). The crystal structure of the mutant, although consistent with the CD results, exhibit interesting differences from the wild-type protein.

The inter domain orientation in $\triangle \mathrm{C} 6 M t \mathrm{RRF}$ with $\psi$ at $83^{\circ}$, is substantially different from that in the wild-type molecule, but close to that in R31AMtRRF. These two are the only known structures of RRF in which the $\psi$ angle is acute. Domain 2 as a whole also appears to undergo a rotation as indicated by the changes in $\chi$ by $24^{\circ}$ (figure 5 and table 4 ). The structure of the two domains themselves, however, remains unaltered. The r.m.s. deviations in $\mathrm{C} \alpha$ positions on superposition are $0.70 \AA$ for domain 1 (sans the six C-terminal residues) and $0.40 \AA$ for domain 2 . Interestingly, however, the structure of the hinge region undergoes a substantial change on the deletion of the C-terminal residues, although they are not part of the hinge. The change is particularly evident in the 27-33 stretch in the hinge. This is not surprising as this stretch interacts with the C-terminal region. In particular, the $\operatorname{Arg} 31 \ldots$ Glu 184 salt bridge is abolished in the mutant as Glu184 is a deleted residue. In fact, in the wild-type protein, the $\mathrm{C}$-terminal region presses against the 27-33 stretch (figure 6). The deletion of the six $\mathrm{C}$-terminal residues appears to facilitate changes in the mutual orientation of the two domains in the mutant. As shown in the figure 6 , a hinge conformation as in the mutant would have led to unacceptable steric clashes in the wild-type protein.

The structures of $\Delta \mathrm{C} 6 M t \mathrm{RRF}$ and $\mathrm{R} 31 \mathrm{~A} M t \mathrm{RRF}$ aid the dissection of the roles of the R31...E184 interaction and the entire C-terminal element on the structure of MtRRF. In wild-type $M t \mathrm{RRF}, \mathrm{R} 31$ at the hinge makes an interaction with E184 at the C-terminus. Removal of the C-terminus in $\Delta \mathrm{C} 6 M t \mathrm{RRF}$ results in the emergence of space behind hinge1 and it moves towards the space left by C-terminus deletion,

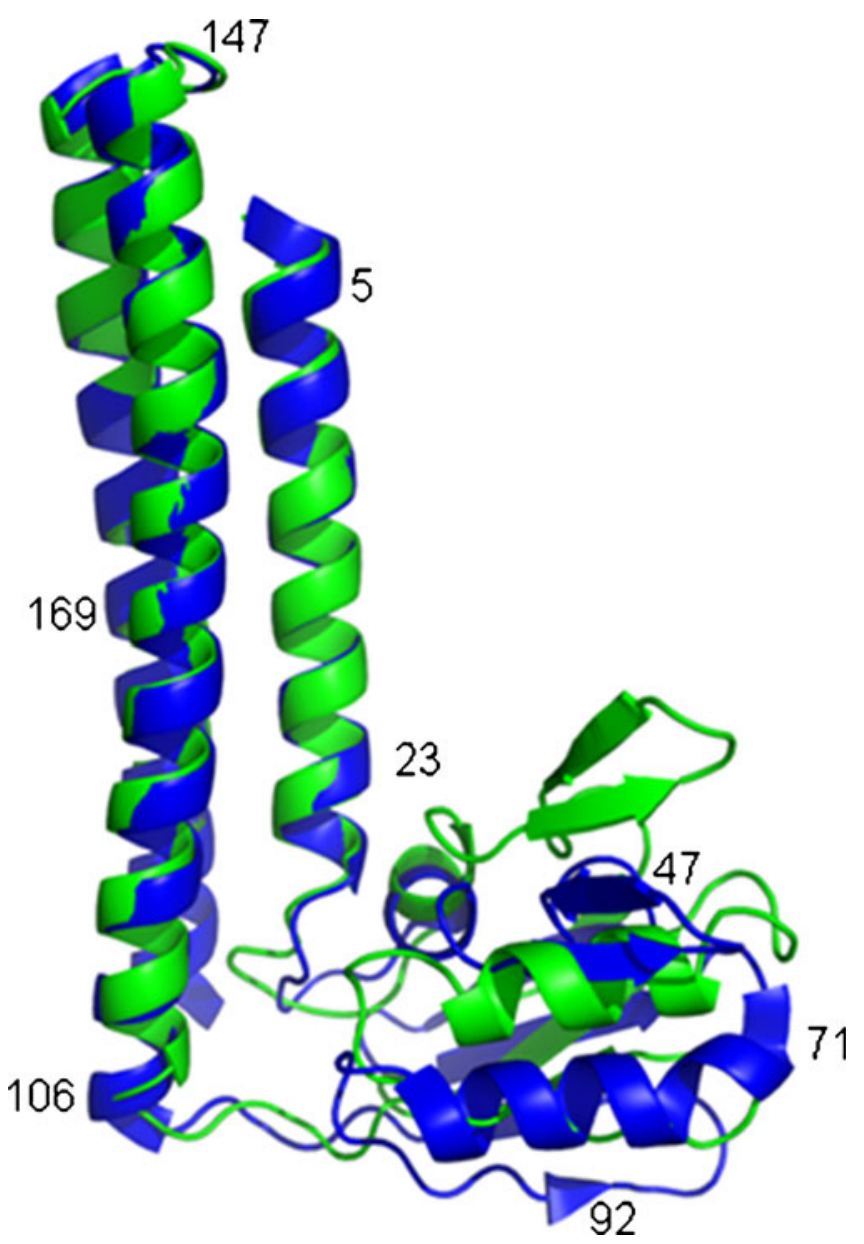

Figure 5. Superposition of $\Delta C 6 M t R R F$ (green) on $M t R R F$ (blue).

resulting in the upward movement of domain 2. In R31A, the presence of $\mathrm{C}$-terminus does not allow the hingel to move towards it, so it moves laterally resulting in a $\varphi$ angle of $-20^{\circ}$ unlike in $\triangle \mathrm{C} 6 M t \mathrm{RRF}$ which has a $\varphi$ value of $0^{\circ}$ (figure 7). Thus the C-terminus either by its presence or absence influences the hinge conformation and thereby controls the domain movement in RRF.

\subsection{RRF structure in the presence of detergent}

In the crystals of wild-type $E c$ RRF grown in the presence of a detergent, decyl- $\beta$-D-maltopyranoside, the two arms of the molecule opens up with a $\psi$ angle of $152^{\circ}$ with the detergent molecule stuck in between the two (Kim et al. 2000). Substantial changes occur in $\varphi$ and $\chi$ as well. Crystals of $E c$ RRF grown in the absence of the detergent have not been reported. However, the structure of an R132G mutant is available (Nakano et al. 2002). The arginine is on the surface of the 


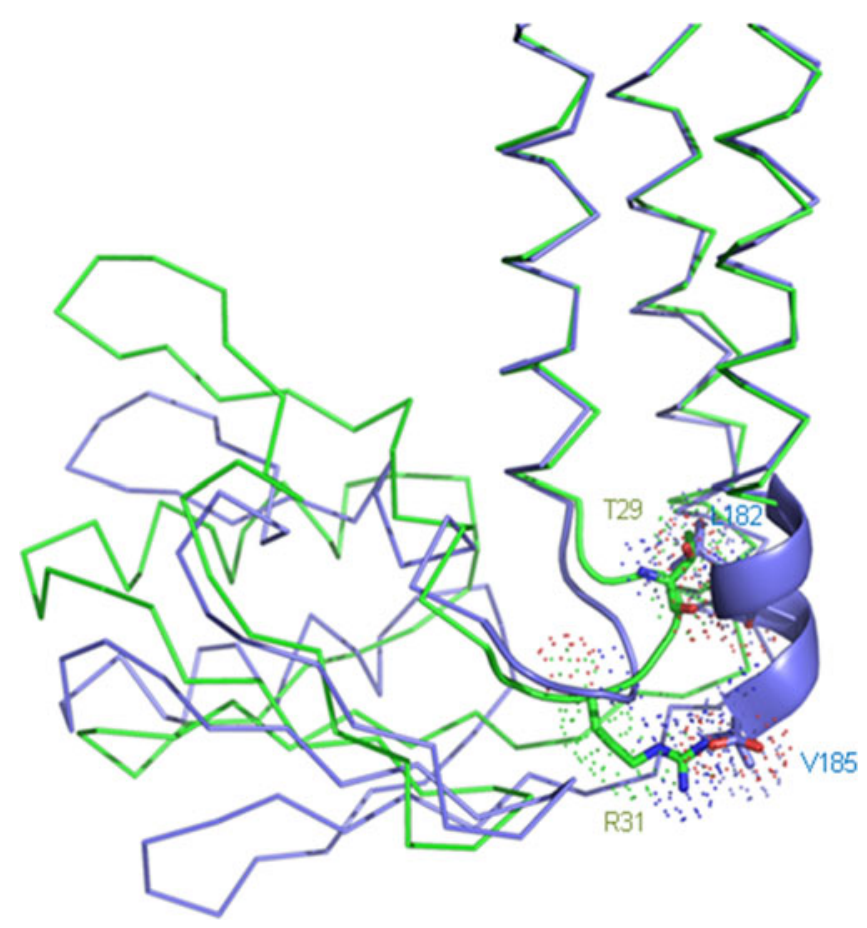

Figure 6. Hinge conformation in $M t R R F$ (blue) and $\Delta C 6 M t R R F$ (green). The dotted region indicates steric clashes between specific residues in the hinge in the mutant and the $\mathrm{C}$-terminal helical segment of the wild-type protein.

molecule. Therefore, this mutation is unlikely to affect the structure of the molecule. The mutant has values of the three

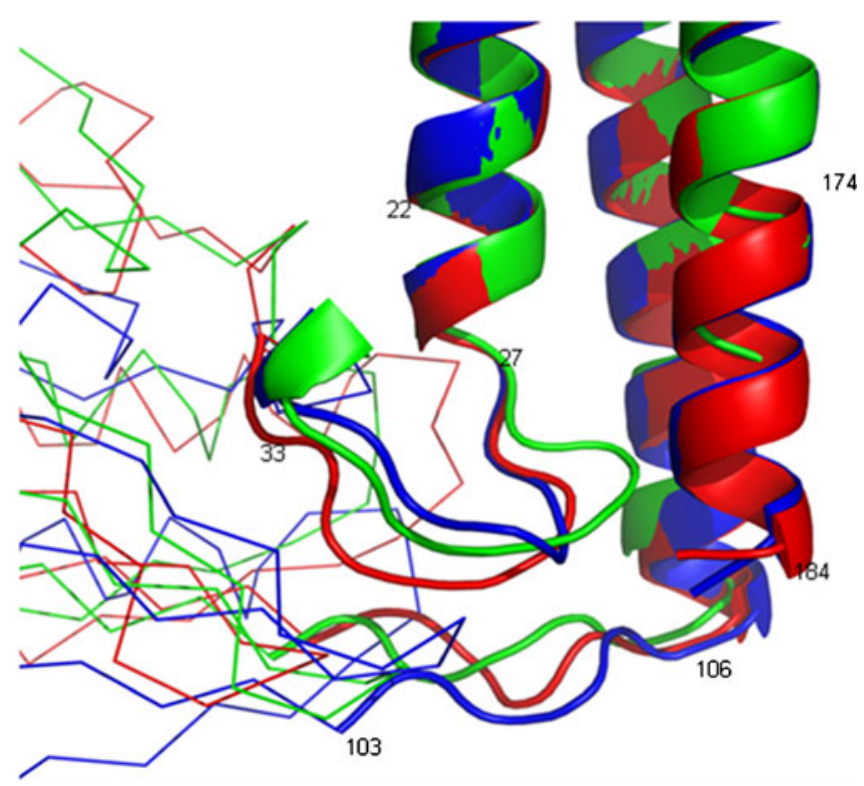

Figure 7. Hinge conformation in $M t \mathrm{RRF}$ (blue), $\Delta \mathrm{C} 6 M t \mathrm{RRF}$ (green) and R31AMtRRF (red). angles comparable to those in $M t R R F$. Thus the opening up of $E c R R F$ is likely to have been caused by the binding of the detergent molecule in between the two arms. It turns out that $M t$ RRF does not open up in the presence of the detergent. The r.m.s. deviations in C $\alpha$ positions on domain-wise superposition also remain low. Clear interpretable density was seen in the structure of MtRRF soaked in the detergent, but it was located at an interstitial position in between two molecules in the crystal structure. Interestingly, perhaps on account of the presence of the hydrophobic ambience created by the detergent in the medium, all the three salt bridges, with reasonable N...O distances, exist in the MtRRF molecule.

Results of the X-ray analysis of crystals grown in the presence of the detergent appeared to indicate that $E c \mathrm{RRF}$ lends itself to opening up of the two arms more easily than $M t$ RRF does. In wild-type $E c R R F$, the detergent occupies a hydrophobic cleft on the surface formed by the residues of hinge region and domain 1 (figure 8a). This cleft is long and continuous and accommodates the alkyl chain of the detergent. The nine residues that interact with the detergent in the $E c R R F$ are highly conserved between $E c$ RRF and $M t R R F$ (5/9 are identical and 4 are similar). Therefore, there is no reason why MtRRF with an obtuse $\psi$ angle cannot accommodate a detergent molecule as EcRRF does. However, the hydrophobic region splits into three when the molecule is L-shaped with $\psi$ nearly $90^{\circ}$ as in the structures of R132GEcRRF and MtRRF. On examining the structure of the R132G mutant of EcRRF, where the molecule is L-shaped and detergent is not bound at the hinge region, the hydrophobic cleft on which the detergent is bound in the wild-type $E c R R F$ is not present (figure $8 b$ ). During domain movement and conformational sampling by $E c$ RRF in solution, the complementary hydrophobic cleft could momentarily form on the surface of $E c R R F$, allowing the detergent to bind there and freeze the domain orientation. In the case of a

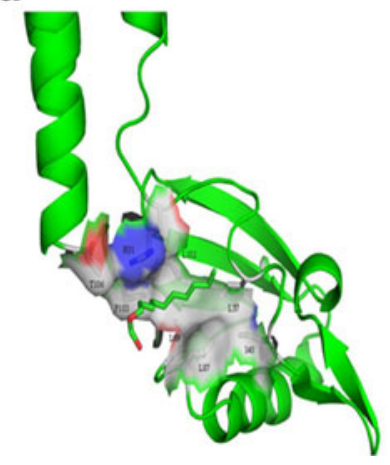

b

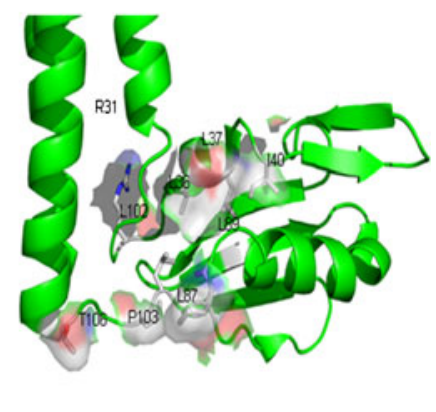

Figure 8. (a)Wild-type $E c R R F$ with bound detergent at a hydrophobic cleft. The hydrophobic patch is indicated. (b) The hydrophobic patches in R132G EcRRF. 
$M t$ RRF, where there are three salt bridges that constrain the relative domain movement, the domains might not even momentarily open up sufficiently to form the continuous hydrophobic cleft. Thus, comparison of structures of detergent bound $E c \mathrm{RRF}, \mathrm{R} 132 \mathrm{G} E c \mathrm{RRF}$ and $M t \mathrm{RRF}$, suggests that detergent binding in $E c R R F$ represents a case of conformational selection. It also suggests that $M t \mathrm{RRF}$ is conformationally more rigid than $E c \mathrm{RRF}$.

\subsection{Displacement parameters as indicators of flexibility}

Individual $\mathrm{B}$ values, which are associated with atoms along with positional coordinates $\mathrm{x}, \mathrm{y}, \mathrm{z}$ in each refined crystal structure, are indicators of the slightly different positions each atom occupies and its thermal vibration amplitudes. Librational parameters obtained from TLS refinement provide information on the oscillatory movements of specified groups about their principal axes (Schoemaker and Trueblood 1968; Winn et al. 2001). In the structures considered here, the three helices are roughly parallel to the axis of domain 1 (Axis 1) defined as the line joining the centres of mass of domain 1 and the hinge region. The axis of domain 2 (Axis 2) is likewise defined as the line joining the centre of mass of the hinge with that of domain 2. The direction perpendicular to the two domain axes is takes as Axis 3.

Magnitudes of libration of each helix in domain 1 about the three principal axes were carefully examined. In every case, the principal axis about which the magnitude is the highest is roughly parallel to the domain axis. The libration about the other two principal axes is often very small, most often less than $10 \%$ of that about the axis nearly parallel to the domain axis. Thus, the motion of the three helices, and therefore that of domain 1 as a whole, can be described primarily as libration about the domain axis. It also turns out that the magnitude is the highest for all the three helices in R31AMtRRF.

The collective movement of domain 2 presents a more complex picture, as can be seen from table 5, which lists the parameters that describe the libration of the domain in the different structures. In all the cases, the major axis of libration is roughly parallel to Axis 2, the angle between the two varying between 16 and $35^{\circ}$. In the wild-type structure and in R39GMtRRF and R109AMtRRF, the minor axis of the ellipsoid is zero or nearly so. The median axis in the three structures, which is less in magnitude than half the respective major axis, is roughly parallel to Axis 3, the angle between the two axes varying from 12 to $32^{\circ}$. Thus, roughly speaking, the motion of domain 2 in the three structures involves primarily variation in $\chi$ and to a lesser extent variation in $\psi$. In $\Delta \mathrm{C} 6 M t \mathrm{RRF}$ and R31AMtRRF also, the main component of motion involves variation of $\chi$, but the other two components cannot be described, even roughly, in terms of variation in the other two angles. Thus the movement of the
Table 5. Angle between principal axes of libration and molecular Axes 1, 2 and 3

\begin{tabular}{|c|c|c|c|c|c|}
\hline \multirow[t]{2}{*}{ S. No. } & \multirow[t]{2}{*}{ Structure } & \multicolumn{3}{|c|}{$\begin{array}{l}\text { Angle between the } \\
\text { principal axis of } \\
\text { libration of domain } \\
2 \text { and molecular axis } \\
\text { (degrees) }\end{array}$} & \multirow[t]{2}{*}{$\begin{array}{l}\text { Mean-square } \\
\text { displacement } \\
\text { about axes } \\
\left(\operatorname{deg}^{2}\right)\end{array}$} \\
\hline & & $\begin{array}{c}\text { Axis } \\
1\end{array}$ & $\begin{array}{c}\text { Axis } \\
2\end{array}$ & $\begin{array}{c}\text { Axis } \\
3\end{array}$ & \\
\hline \multirow[t]{3}{*}{1} & \multirow[t]{3}{*}{$M t \mathrm{RRF}$} & 82 & 16 & 91 & 6.5 \\
\hline & & 59 & 80 & 32 & 2.3 \\
\hline & & 33 & 76 & 58 & 0.3 \\
\hline \multirow[t]{3}{*}{2} & \multirow[t]{3}{*}{ R39GMtRRF } & 104 & 23 & 92 & 13.0 \\
\hline & & 65 & 97 & 26 & 5.0 \\
\hline & & 30 & 68 & 115 & 0.0 \\
\hline \multirow[t]{3}{*}{3} & \multirow[t]{3}{*}{ R109AMtRRF } & 104 & 23 & 92 & 14.9 \\
\hline & & 85 & 101 & 12 & 4.0 \\
\hline & & 14 & 68 & 96 & 0.0 \\
\hline \multirow[t]{3}{*}{4} & \multirow[t]{3}{*}{$\mathrm{R} 31 \mathrm{~A} M t \mathrm{RRF}$} & 100 & 27 & 67 & 13.4 \\
\hline & & 32 & 114 & 66 & 5.9 \\
\hline & & 60 & 102 & 33 & 2.3 \\
\hline \multirow[t]{3}{*}{5} & \multirow[t]{3}{*}{$\Delta \mathrm{C} 6 M t \mathrm{RRF}$} & 51 & 36 & 98 & 3.8 \\
\hline & & 50 & 110 & 50 & 1.7 \\
\hline & & 65 & 62 & 42 & 0.6 \\
\hline
\end{tabular}

See text for details.

domains in these two mutants is somewhat different from that in the other three structures. It also turns out that among the five structures, the magnitude of libration is the lowest in $\Delta \mathrm{C} 6 M t \mathrm{RRF}$ and the highest in R31AMtRRF.

\section{Discussion}

The crystal structures of the mutants and their genetic analysis provide interesting insights into the relation between the plasticity of the MtRRF molecule and its biological activity. All the point mutants retain activity with its homologous EF-G (MtEF-G) although a double mutant exhibits somewhat lower activity. Thus, it would appear that the overall biological function of the protein is comparatively unaffected by the presence or absence of interdomain salt bridges. The mutant studied which is totally inactive for its RRF function is $\Delta \mathrm{C} 6 M t \mathrm{RRF}$. Deletion of the $\mathrm{C}$-terminal residues leads to substantial changes in the hinge region with considerable distortion of the molecule in terms of interdomain orientation. The point mutant R31AMtRRF, in which the R31...E184 salt bridge between the hinge and the $\mathrm{C}$-terminal region is abolished, also has changes in the hinge region and the consequent distortion of the molecule. 
However, the changes in the hinge region appears to have opposite effects in the two mutants in terms of mobility with particular reference to the movement of domain 2, which protrudes out of the RRF bound ribosome, interacts with EF-G and causes disorder in H69 of ribosome intersubunit bridge $\mathrm{B} 2 \mathrm{a}$ as a prelude to disassembly. The libration of the domain is substantially lower in magnitude in $\Delta \mathrm{C} 6 M t \mathrm{RRF}$ compared to that in the wild-type molecule, while it is much higher in R31AMtRRF. This increased mobility does not affect the activity of R31AMtRRF with MtEF-G. The loss of activity resulting from the deletion of the C-terminal residues in $\Delta \mathrm{C} 6 M t \mathrm{RRF}$ could partly be due to the decreased mobility of domain 2 . It is also possible that it is partly or wholly on account of the impairment of appropriate interactions with EF-G.

Presumably the abolition of one or the other of two interdomain salt bridges as in R39GMtRRF or R109AMtRRF or both as in the double mutant R39G.R109AMtRRF, is not enough to make the $M t R R F$ molecule to function with $E c$ EF-G. However, as the librational parameters indicate, abolition of the salt bridge between the hinge and the $\mathrm{C}$-domain, as in R31AMtRRF, leads to substantial increased flexibility of the molecule. It appears that it is this flexibility that enables R31AMtRRF to at least weakly function with $E c$ EF-G. This further brings out the importance of molecular flexibility in the function of RRF.

\section{PDB accession codes}

The atomic coordinates and structure factors of all $M t \mathrm{RRF}$ structures reported here have been deposited in the Research Collaboratory for Structural Bioinformatics PDB with the following accession codes: R39GMtRRF, 4KAW; R109AMtRRF, 4KB2; R31AMtRRF, 4KB4; $\Delta \mathrm{C} 6 M t \mathrm{RRF}$, $4 \mathrm{KC} 6$; and $M t \mathrm{RRF}$ grown in presence of detergent, 4KDD.

\section{Acknowledgements}

The work has been supported by the Department of Biotechnology, India. X-ray intensity data were collected at the DST supported facility for protein X-ray crystal structure determination and protein design. Part of the computations was carried out at the Interactive Graphics Facility supported by the DBT. MV is the Albert Einstein Professor of the Indian National Science Academy.

\section{References}

Arora A, Chandra NR, Das A, Gopal B, Mande SC, Prakash B, Ramachandran R, Sankaranarayanan R, et al. 2011 Structural biology of Mycobacterium tuberculosis proteins: the Indian efforts. Tuberculosis (Edinb) 91 456-468
Brünger AT, Adams PD, Clore GM, DeLano WL, Gros P, GrosseKunstleve RW, Jiang JS, Kuszewski J, et al. 1998 Crystallography \& NMR system: A new software suite for macromolecular structure determination. Acta Crystallogr. D Biol. Crystallogr. 54 905-921

Chetnani B, Kumar P, Surolia A and Vijayan M 2010 M. tuberculosis pantothenate kinase: dual substrate specificity and unusual changes in ligand locations. J. Mol. Biol. 400 $171-185$

Emsley P and Cowtan K 2004 Coot: model-building tools for molecular graphics. Acta Crystallogr. D Biol. Crystallogr. 60 2126-2132

Fujiwara T, Ito K, Nakayashiki T and Nakamura Y 1999 Amber mutations in ribosome recycling factors of Escherichia coli and Thermus thermophilus: evidence for C-terminal modulator element. FEBS Lett 447 297-302

Hirashima A and Kaji A 1972 Factor dependent release of ribosomes from messenger RNA. Requirement of two heat stable factors. J. Mol. Biol. 65 43-58

Hirokawa G, Demeshkina N, Iwakura N, Kaji H and Kaji A 2006 The ribosome-recycling step: consensus or controversy? Trends Biochem. Sci. 31 143-149

Janosi L, Mottagui-Tabar S, Isaksson LA, Sekine Y, Ohtsubo E, Zhang S, Goon S, Nelken S, Shuda M and Kaji A 1998 Evidence for in vivo ribosome recycling, the fourth step in protein biosynthesis. EMBO J. 17 1141-1151

Kaushal PS, Talawar RK, Krishna PD, Varshney U and Vijayan M 2008 Unique features of the structure and interactions of mycobacterial uracil-DNA glycosylase: structure of a complex of the Mycobacterium tuberculosis enzyme in comparison with those from other sources. Acta Crystallogr. D Biol. Crystallogr. 64 $551-560$

Kim KK, Min K and Suh SW 2000 Crystal structure of the ribosome recycling factor from Escherichia coli. EMBO J. $192362-$ 2370

Krishna R, Prabu JR, Manjunath GP, Datta S, Chandra NR, Muniyappa K and Vijayan M 2007 Snapshots of RecA protein involving movement of the $\mathrm{C}$-domain and different conformations of the DNA-binding loops: crystallographic and comparative analysis of 11 structures of Mycobacterium smegmatis RecA. J. Mol. Biol. 367 1130-1144

Nakano H, Uchiyama S, Yoshida T, Ohkubo T, Kato H, Yamagata Y and Kobayashi Y 2002 Crystallization and preliminary X-ray crystallographic studies of a mutant of ribosome recycling factor from Escherichia coli, Arg132Gly. Acta Crystallogr. D Biol. Crystallogr. 58 124-126

Prabu JR, Thamotharan S, Khanduja JS, Chandra NR, Muniyappa $\mathrm{K}$ and Vijayan M 2009 Crystallographic and modelling studies on Mycobacterium tuberculosis RuvA Additional role of RuvBbinding domain and inter species variability. Biochim. Biophys. Acta 1794 1001-1009

Rao AR and Varshney U 2001 Specific interaction between the ribosome recycling factor and the elongation factor $\mathrm{G}$ from Mycobacterium tuberculosis mediates peptidyl-tRNA release and ribosome recycling in Escherichia coli. EMBO J $202977-$ 2986

Schoemaker V and Trueblood KN 1968 On the rigid-body motion of molecules in crystals. Acta Crystallogr. B23 63-76 
Rao AR and Varshney U 2002 Characterization of Mycobacterium tuberculosis ribosome recycling factor (RRF) and a mutant lacking six amino acids from the C-terminal end reveals that the C-terminal residues are important for its occupancy on the ribosome. Microbiology 148 3913-3920

Roy S, Saraswathi R, Chatterji D and Vijayan M 2008 Structural studies on the second Mycobacterium smegmatis Dps: invariant and variable features of structure, assembly and function. J. Mol. Biol. 375 948-959

Saikrishnan K, Kalapala SK, Varshney U and Vijayan M 2005 Xray structural studies of Mycobacterium tuberculosis RRF and a comparative study of RRFs of known structure. Molecular plasticity and biological implications. J. Mol. Biol. 345 29-38

Saikrishnan K, Kalapala SK, Bidya Sagar M, Rao AR, Varshney U and Vijayan M 2004 Purification, crystallization and preliminary X-ray studies of Mycobacterium tuberculosis RRF. Acta Crystallogr. D Biol. Crystallogr. 60 368-370

Selmer M, Al-Karadaghi S, Hirokawa G, Kaji A and Liljas A 1999 Crystal structure of Thermotoga maritima ribosome recycling factor: a tRNA mimic. Science 286 2349-2352

Selvaraj M, Roy S, Singh NS, Sangeetha R, Varshney U and Vijayan M 2007 Structural plasticity and enzyme action: crystal structures of Mycobacterium tuberculosis peptidyl-tRNA hydrolase. J. Mol. Biol. 372 186-193

Seshadri A, Singh NS and Varshney U 2010 Recycling of the posttermination complexes of Mycobacterium smegmatis and
Escherichia coli ribosomes using heterologous factors. J. Mol. Biol. $401854-865$

Seshadri A and Varshney U 2006 Mechanism of recycling of posttermination ribosomal complexes in eubacteria: a new role of initiation factor 3. J. Biosci. 31 281-289

Storoni LC, McCoy AJ and Read RJ 2004 Likelihood-enhanced fast rotation functions. Acta Crystallogr. D Biol. Crystallogr. 60 432-438

Terwilliger TC, Park MS, Waldo GS, Berendzen J, Hung LW, Kim CY, Smith CV, Sacchettini JC, et al. 2003 The TB structural genomics consortium: a resource for Mycobacterium tuberculosis biology. Tuberculosis (Edinb) 83 223-249

Toyoda T, Tin OF, Ito K, Fujiwara T, Kumasaka T, Yamamoto M, Garber MB and Nakamura Y 2000 Crystal structure combined with genetic analysis of the Thermus thermophilus ribosome recycling factor shows that a flexible hinge may act as a functional switch. RNA 6 1432-1444

Vijayan M 2005 Structural biology of mycobacterial proteins: the Bangalore effort. Tuberculosis (Edinb) 85 357-366

Winn MD, Isupov MN, Murshudov GN 2001 Use of TLS parameters to model anisotropic displacements in macromolecular refinement. Acta Crystallogr. D Biol. Crystallogr. 57 122-133

Winn MD, Ballard CC, Cowtan KD, Dodson EJ, Emsley P, Evans PR, Keegan RM, Krissinel EB, et al. 2011 Overview of the CCP4 suite and current developments. Acta Crystallogr. D Biol. Crystallogr. 67 235-242

MS received 17 July 2013; accepted 10 September 2013

Corresponding editor: SEyed E Hasnain 